\title{
Optical emission spectra of high current and high voltage generated arcs representing lightning
}

Cite as: Appl. Phys. Lett. 114, 164103 (2019); https://doi.org/10.1063/1.5092875

Submitted: 15 February 2019. Accepted: 07 April 2019 . Published Online: 26 April 2019

D. Mitchard (D), P. Widger (D), D. Clark (D), D. Carr (D), and A. Haddad (D)
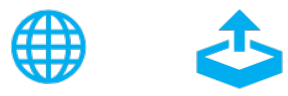

\section{ARTICLES YOU MAY BE INTERESTED IN}

Experimental demonstration of energy harvesting from the sky using the negative illumination effect of a semiconductor photodiode

Applied Physics Letters 114, 161102 (2019); https://doi.org/10.1063/1.5089783

Laser-driven semiconductor switch for generating nanosecond pulses from a megawatt gyrotron

Applied Physics Letters 114, 164102 (2019); https://doi.org/10.1063/1.5093639

A gas flow meter with linear sensitivity based on freely-suspended nanofilms of smectic liquid crystal

Applied Physics Letters 114, 163705 (2019); https://doi.org/10.1063/1.5083967

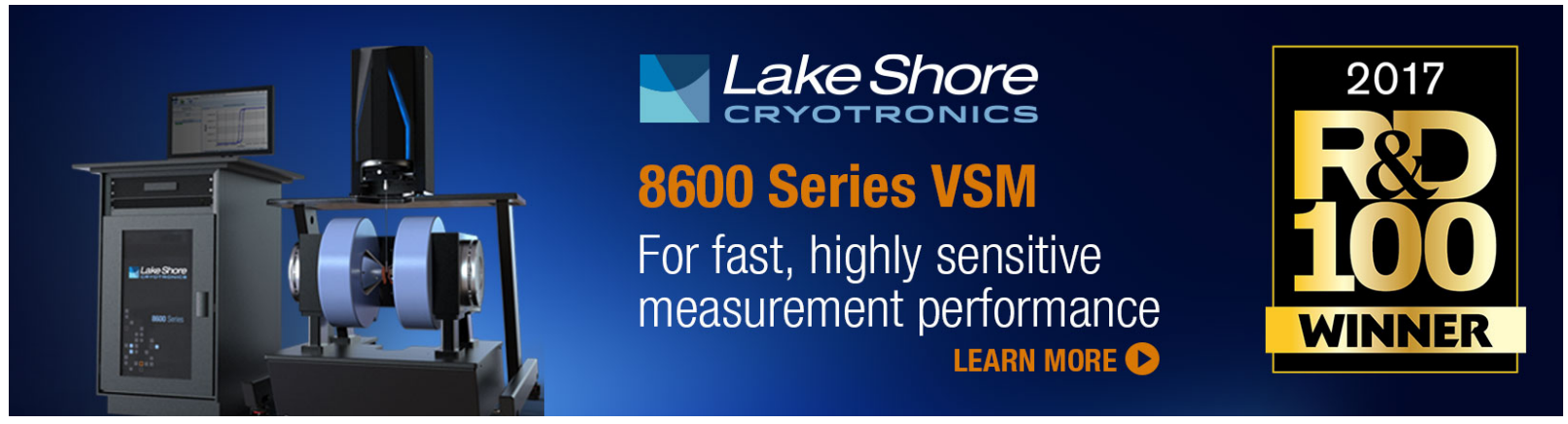




\title{
Optical emission spectra of high current and high voltage generated arcs representing lightning
}

\author{
Cite as: Appl. Phys. Lett. 114, 164103 (2019); doi: 10.1063/1.5092875 \\ Submitted: 15 February 2019 - Accepted: 7 April 2019 . \\ Published Online: 26 April 2019
}

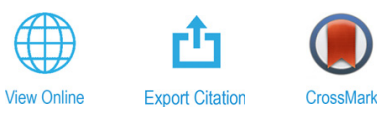

D. Mitchard, ${ }^{\text {a) }}$ (D) P. Widger, (D) D. Clark, (D) D. Carr, (D) and A. Haddad (D)

\begin{abstract}
AFFILIATIONS
Advanced High Voltage Engineering Research Centre, School of Engineering, Cardiff University, Cardiff CF24 3AA, Wales, United Kingdom
\end{abstract}

a) Author to whom correspondence should be addressed: mitcharddr@cardiff.ac.uk

\begin{abstract}
A recently developed spectrographic technique was used for the comparison of observed optical emission spectra from self-breakdown in air of high current and high voltage impulse arcs which are often used to represent the respective components of a natural lightning strike for a variety of lightning studies. These components are separated as it is practically impossible to simultaneously generate both in a single arc within the laboratory. The high current aspect is typically related to the amount of damage a lightning arc does to an impacted object and can be used to characterize conductivity properties, whereas the high voltage aspect is typically related to the attachment mechanism of lightning to an object when creating a path to ground and can be used to characterize insulation properties. The spectra of a range of high current arcs up to $100 \mathrm{kA}$ and high voltage arcs up to $120 \mathrm{kV}$ across a $14 \mathrm{~mm}$ air-gap between a pair of hemispherical tungsten electrodes were investigated and compared with the natural lightning reference spectrum reconstructed from the literature. All three spectra were found to present similar trends, but the high voltage arc had notably lower blackbody radiation, indicating a less energetic and cooler arc. Further, whereas atomic lines from oxygen and nitrogen were clearly present, an atomic line from argon was not prominent, indicating that the bulk energy delivered into the air was in the range of 1400-1520 kJ/mol. The high current arc, much like natural lightning, delivered a bulk energy greater than $1520 \mathrm{~kJ} / \mathrm{mol}$.
\end{abstract}

Published under license by AIP Publishing. https://doi.org/10.1063/1.5092875

Lightning is one of the most destructive and unpredictable forces in nature; a natural lightning strike consists of an average current magnitude of $30 \mathrm{kA}$, but can be $200 \mathrm{kA}$ or higher, and potentials on the order of tens of gigavolts within a couple of hundred microseconds. There has been extensive scientific interest in studying such events, and from the 1960s, numerous optical emission spectral methods were developed and used to offer a greater insight into lightning mechanisms. This was first achieved using traditional film technology ${ }^{1-4}$ and moved onto digital camera techniques, ${ }^{5-8}$ typically focusing on the ultraviolet to near-infrared wavelength range. Such methods have proved to be successful in deriving characteristics such as arc temperature, pressure, electron density, and energy. ${ }^{9-13}$ However, the inherently random and nonrepeatable nature of natural lightning has made it very difficult to systematically study the phenomenon and to understand lightning interactions and the impact on the surrounding environment. This is particularly relevant in research fields related to the safety and security of vulnerable transportation and the built environment. Reproducing repeatable lightning events within a controlled laboratory environment allows a closer study and better understanding using the same methods, ${ }^{14,15}$ but it is not possible to reproduce the combined high current and high voltage components of a natural lightning strike simultaneously, and so, for laboratory tests, either the high current or the high voltage is reproduced representing an individual lightning impulse component.

High current lightning impulse generators are often designed to replicate the destructive component of a lightning strike characterized by a very large current impulse through a medium or onto a material. It is this component, through the explosive expansion of air, which is largely associated with thunder. Such generators can deliver $200 \mathrm{kA}$ or more within a duration of around $300 \mu$ s at voltages in tens of kilovolts, such as those reported in Ref. 16. These impulse parameters are important when a clear path to ground cannot be achieved, meaning that an object struck by lightning must absorb and distribute the full energy of the strike. Such energy levels are of notable significance for airborne commercial aircraft, which needs to be designed to conduct worst-case lightning currents, whilst in flight, from the entry to exit points, with minimal surface and bulk material damage and no disruption to flight control and fuel tanks. 
Alternatively, high voltage generators are often designed to replicate the high voltage surges caused by a lightning strike through a medium or onto an insulating material. It is this component, through the development of streamers and leaders, which is largely associated with the forking of lightning arcs. Such generators can deliver hundreds, even thousands, of kilovolts in less than $100 \mu$ s at currents in the range of kiloamp, such as described in Ref. 17. These characteristics are important for the safety and protection of infrastructure during a lightning strike, which is of notable significance for the built environment, such as overhead electricity lines or electric rail networks, where the lightning surges can travel along the network and cause breakdowns of insulation systems, leading to equipment damage and injury to personnel.

In this work, a study was designed to investigate the optical emission spectra of laboratory generated high current and high voltage lightning arcs under very similar experimental conditions, both of which were then compared with the spectrum of a natural lightning arc reconstructed from the literature. For the high current lightning arc test, the 18/40 $\mu$ s impulse D waveform from the EUROCAE ED84 aerospace standard ${ }^{18}$ was chosen for peak currents of $40,60,80$, and $100 \mathrm{kA}$. For the high voltage lightning arc test, the $1.2 / 50 \mu$ s impulse standard waveform from the IEC 60060 standard on the high voltage measurement and testing techniques ${ }^{19}$ was chosen with peak voltages of $60,80,100$, and $120 \mathrm{kV}$. The natural lightning reference was reconstructed from Ref. 2, as had been done in Ref. 14, which used a variety of traditional photographic films across multiple overlapping wavelength ranges with varying light intensity and background levels. However, as light intensity across each film could not be normalized, it only acts as a qualitative comparison for the position and approximate relative intensity of the atomic lines.

The experiment was designed such that the same test electrode and measurement apparatus were used with the generated lighting waveform connected to either a high current or high voltage source. A pair of $60 \mathrm{~mm}$ diameter hemispherical polished tungsten vertically aligned electrodes were separated by an air gap of $14 \mathrm{~mm}$ with the top electrode connected to the energization terminal and the bottom electrode connected to ground; the distance between the electrodes was designed to ensure that self-breakdown would occur at the lowest generator charging voltage of $22 \mathrm{kV}$, i.e., the $40 \mathrm{kA}$ peak high current lightning arc test. Tungsten was chosen as the electrode material as it is a material which can better withstand the numerous lightning strikes required during experimentation with minimal damage but, more importantly for this study, for having relatively few spectral atomic lines across the ultraviolet to infrared range compared to other metals. The electrodes were securely mounted within an electromagnetically shielded light-tight chamber, such that the only light present was that from the arc, which also contained an optic fiber used to transport the light to an external spectrograph. The spectrograph consisted of a slit, which was adjusted to $100 \mathrm{~nm}$ to control the signalto-noise ratio of incoming light and spectral atomic line sharpness, and a 1200 line $/ \mathrm{mm}$ grating giving a $0.6 \mathrm{~nm}$ resolution across a $570 \mathrm{~nm}$ bandwidth. A second grating of 900 line/ $\mathrm{mm}$ was also used giving a $0.2 \mathrm{~nm}$ resolution across a $140 \mathrm{~nm}$ bandwidth with the resulting images stitched together to create a broader spectrum as described in Ref. 20. A spectral range of $450 \mathrm{~nm}-900 \mathrm{~nm}$ was chosen as this is the typical range used in numerous previous studies, with the spectrograph being calibrated beforehand using a mercury-argon light source.
During the experiments, the shutter on the spectrograph was left open for $5 \mathrm{~s}$ to ensure that all light from the arc was captured, with the arc occurring roughly in the middle of the exposure. Any data which contained anomalies, such as cosmic ray spikes, were disregarded, and the experiment was repeated. A schematic of the experimental setup is shown in Fig. 1.

The waveform of each generator was first recorded to ensure compliance with each relevant standard. For the high current generator, a Rogowski coil with an active integrating circuit positioned before the top electrode was used to measure the short circuit current waveform during breakdown, recording the results shown in Fig. 2(a). The corresponding peak charging voltages were set as given in Table I. For the high voltage generator, a voltage divider positioned before the energization electrode was used to measure the open circuit voltage waveform, with the results shown in Fig. 2(b). The corresponding peak currents were measured as given in Table II. Both the high current and high voltage waveforms were found to be in accordance with their relevant standards.

Using the 1200 line/mm spectrograph grating and starting with the high current generator at $40 \mathrm{kA}$, four lightning strikes were carried out with the light from each being recorded by the spectrograph. The spectra were averaged to produce a single spectrum. The current generator was then switched to $60 \mathrm{kA}$, and the experiment was repeated until $100 \mathrm{kA}$ was reached. The results are shown in Fig. 3(a). The high voltage generator was then used, and the above steps were repeated for $60 \mathrm{kV}-120 \mathrm{kV}$. The results are shown in Fig. 3(b). It can be seen that, as both the high current and the high voltage peak magnitude increase, the blackbody radiation level and intensity of the atomic lines also increase. This is as expected as, in both the cases, the amount of energy released is also increasing. It can also be seen that the blackbody radiation level is higher for the high current arc in comparison to the high voltage arc. An accurate measure of either the temperature or the energy cannot be obtained because the data consist of an integrated spectrum across all light during the lifetime of the arc, but the trends can be used to infer a higher temperature and energy. ${ }^{21}$

For a more detailed comparison at a higher atomic line resolution, the 900 line/mm spectrograph grating was selected and a stitching method was used to create a broad spectrum. This was done for the maximum value of each generator of $100 \mathrm{kA}$ and $120 \mathrm{kV}$ as these spectra displayed the most prominent trends. The results are shown in Fig. 4(a), which shows the same trends as Fig. 3. Each spectrum was then converted into an intensity plot and compared with the natural

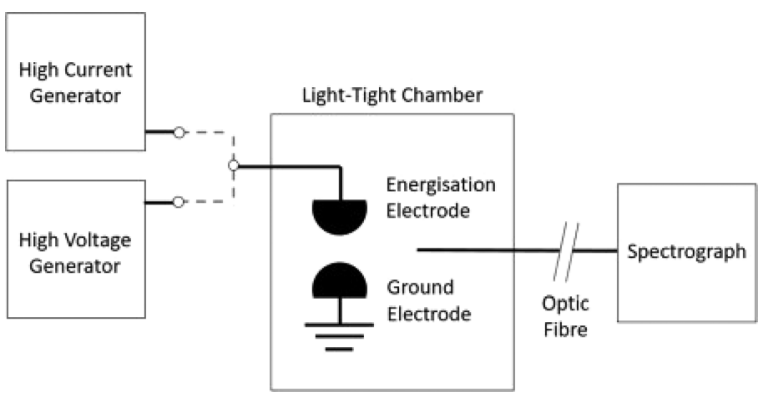

FIG. 1. A schematic of the experimental setup (not to scale) showing the two generators, electrode arrangement, and spectrograph. Either the high current generator or the high voltage generator is connected to the energization electrode at any one time. 

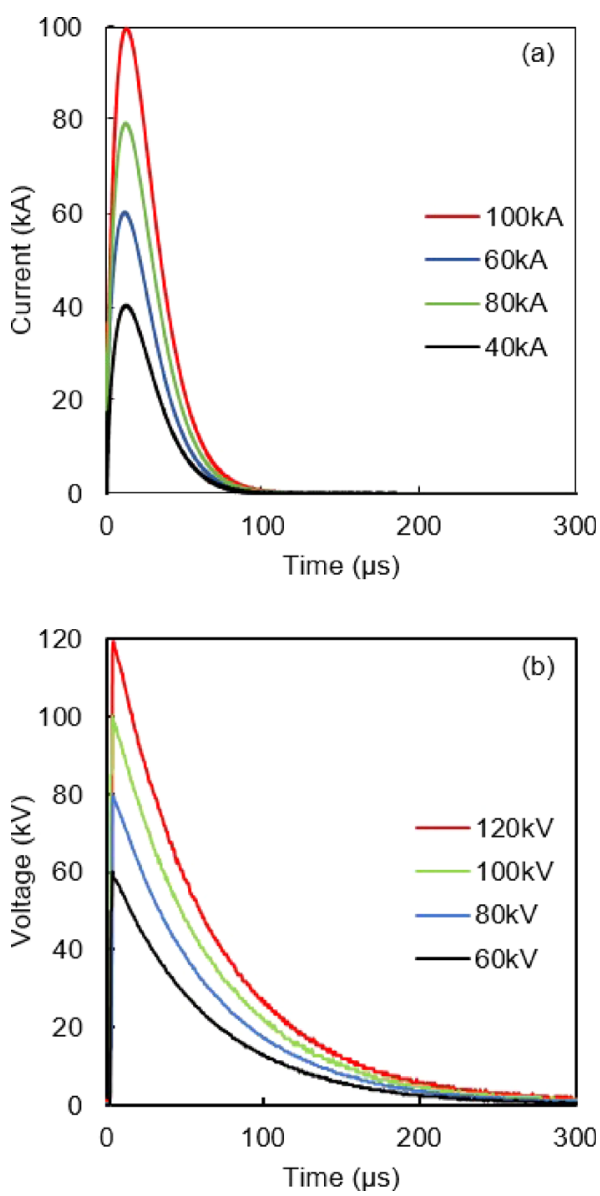

FIG. 2. (a) The measured peak current waveforms for the high current generator complying with the relevant standard. ${ }^{16}$ (b) The measured peak voltage waveforms for the high voltage generator complying with the relevant standard.

TABLE I. The corresponding generator charging voltage for each respective output peak current.

\begin{tabular}{llllc}
\hline \hline Generator charging voltage (kV) & 22 & 32 & 43 & 54 \\
Output peak current (kA) & 40 & 60 & 80 & 100 \\
\hline \hline
\end{tabular}

TABLE II. The corresponding peak current measured for each respective high voltage setting.

\begin{tabular}{lcccc}
\hline \hline Measured peak current $(\mathrm{kA})$ & 2.2 & 2.9 & 3.6 & 6.3 \\
High voltage setting $(\mathrm{kV})$ & 60 & 80 & 100 & 120 \\
\hline \hline
\end{tabular}

lightning reference as shown in Fig. 4(b). Prominent atomic lines have been be identified, all of which are constituents of the air, such as NI, OI, ArI, and helium, with the addition of several lines mostly in the region of 450-600 nm of WI from the electrodes. Higher order ionization states such as NII and OII are not included or discussed here with more comprehensive references being found in Refs. 2 and 22.
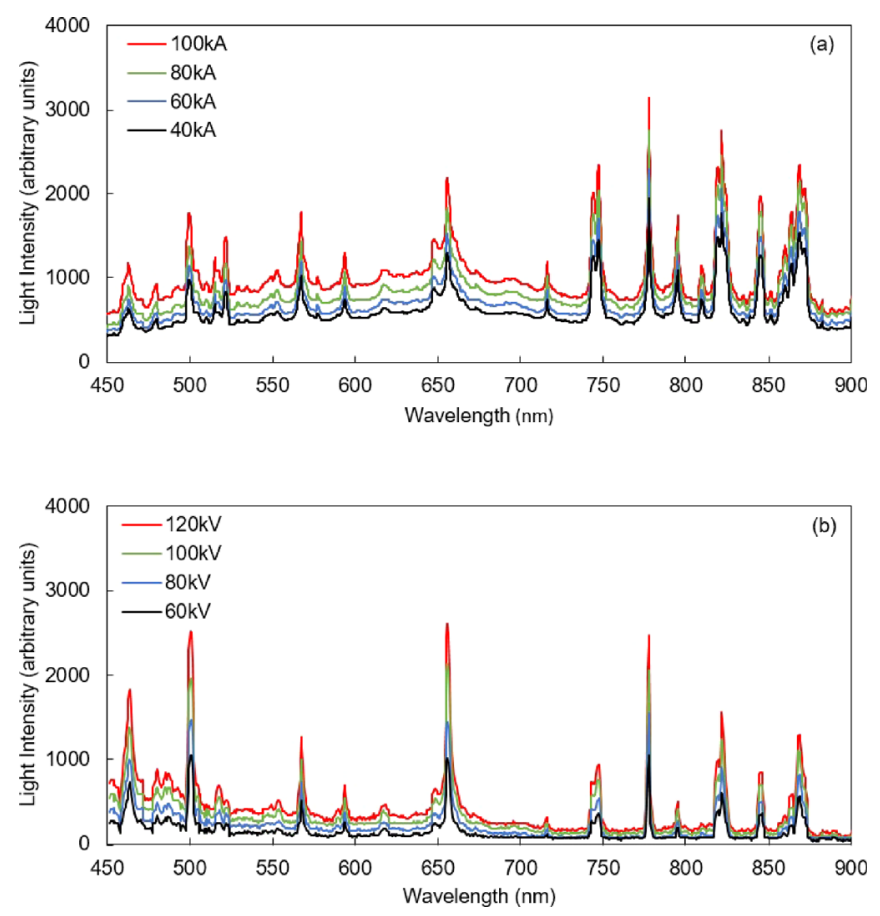

FIG. 3. (a) The spectra for various high current waveforms and (b) high voltage waveforms representing individual components of a natural lightning strike.

The majority of clearly identifiable atomic lines are present in all three spectra as presented in Fig. 4. However, there is a peak between 810 and $812 \mathrm{~nm}$ present in the high current arc and natural lightning reference which is barely visible in the high voltage arc. This wavelength region corresponds to the most prominent argon line in the observed $450-900 \mathrm{~nm}$ range at $811.53 \mathrm{~nm}$, with a second less prominent line very close at $810.37 \mathrm{~nm}$. The lack of these atomic lines in the high voltage spectrum can be explained by considering the first ionization energies of the four observed elements: tungsten, $770 \mathrm{~kJ} / \mathrm{mol}$; oxygen, $1313.9 \mathrm{~kJ} / \mathrm{mol}$; nitrogen, $1402.3 \mathrm{~kJ} / \mathrm{mol}$; and $\operatorname{argon}, 1520.6 \mathrm{~kJ} / \mathrm{mol}$. Argon requires the largest amount of energy to be ionized, and it appears that, although the high voltage arc has sufficient energy to ionize nitrogen, it does not have the energy content required to ionize argon. This suggests that the amount of energy the high voltage arc delivers into the air is between that of nitrogen and argon, i.e., approximately 1400 and $1520 \mathrm{~kJ} / \mathrm{mol}$. As nitrogen lines are always visible across $60-120 \mathrm{kV}$ as seen earlier in Fig. 3, this energy range also applies to those voltages. As argon is present in both the high current and natural lightning references, both the energies delivered into the air by these arcs must be greater than $1520 \mathrm{~kJ} / \mathrm{mol}$. Similarly, as the argon lines are visible across $40-100 \mathrm{kA}$, this must also apply to those arcs.

It is important to note that these values are related to the bulk energy of the lightning arc itself and not the individual energy of the electrons traveling between the electrodes within the arc. For the high current experiments, there is a high flow of charge accelerated across a high electromagnetic field; the probability of individual electrons encountering and ionizing argon atoms within the air was high simply due to the large number of electrons within the arc, and overall, this resulted in a strong atomic line. For the high voltage experiments, 

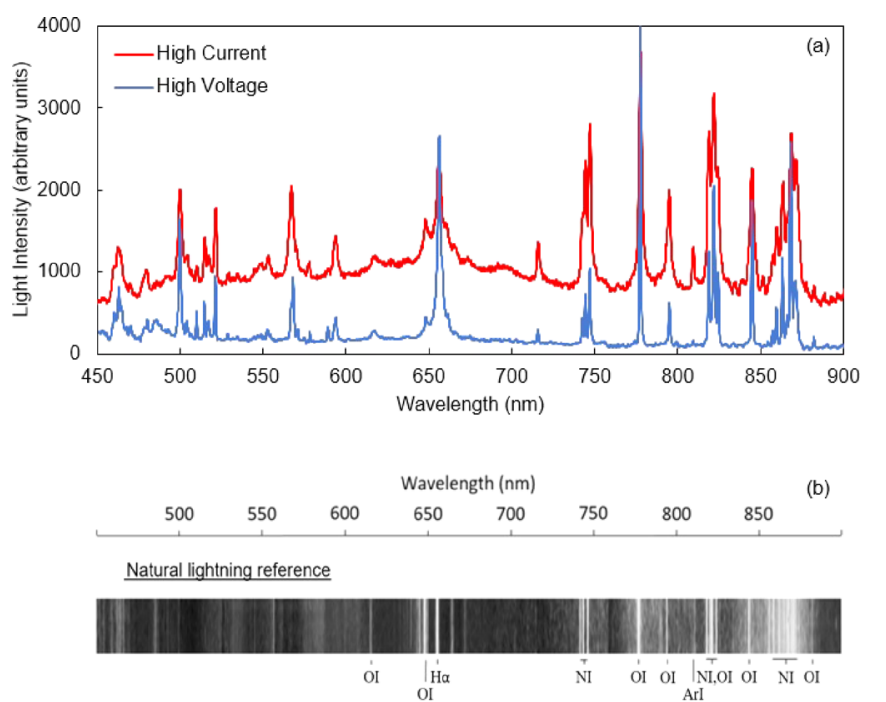

High current generated lightning arc

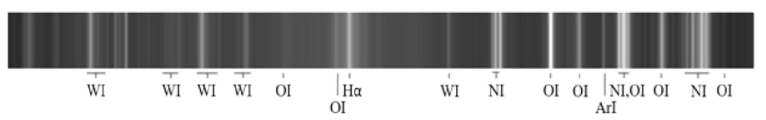

High voltage generated lightning arc

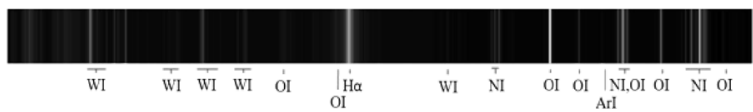

FIG. 4. (a) The spectrum of the generated high current and high voltage lightning components and (b) the corresponding intensity plots compared to the natural lightning reference, ${ }^{2,14}$ with first order element ionization levels labeled.

there was a relatively low flow of charge, but it is accelerated across a higher electromagnetic field. Each individual electron had more kinetic energy than in the previous case, but the probability of encountering and ionizing an argon atom was low due to the small number of electrons within the arc, and overall, this resulted is a weak atomic line.

In summary, the high current and high voltage spectra are comparable to the natural lightning reference, with each presenting a fairly accurate representation for use in laboratory testing to gain knowledge of the lightning phenomenon. Differences lie in the level of blackbody radiation and relative peaks and broadening of atomic lines, indicating that a high voltage arc is less energetic and cooler. Further analysis indicates that the energy a $60-120 \mathrm{kV}$ high voltage arc can deliver into a medium is between 1400 and $1520 \mathrm{~kJ} / \mathrm{mol}$, whereas the energy a $40-100 \mathrm{kA}$ arc can deliver is more than $1520 \mathrm{~kJ} / \mathrm{mol}$, with the latter being most similar to a natural lightning arc.

The authors gratefully acknowledge the financial support provided by the Sêr Cymru National Research Network in Advanced Engineering and Materials (NRN073), Innovate UK via the Aerospace Technology Institute (113037), and EU/Welsh Government funded project, FLEXIS.

\section{REFERENCES}

${ }^{1}$ L. E. Salanave, Science 134, 1395-1399 (1961).

${ }^{2}$ L. Wallace, Astrophys. J. 139, 994 (1963).

${ }^{3}$ R. E. Orville and L. E. Salanave, Appl. Opt. 9(9), 1775-1791 (1970).

${ }^{4}$ L. E. Salanave, Appl. Opt. 10(1), 206-207 (1971).

${ }^{5}$ T. D. Walker and H. J. Christian, in XV International Conference on Atmospheric Electricity, USA (2014).

${ }^{6}$ R. E. Orville, J. Appl. Meteorol. 19(4), 470 (1980).

${ }^{7}$ R. Orville and R. Henderson, J. Atmos. Sci. 41(21), 3180-3187 (1984).

${ }^{8}$ R. E. Orville, J. Atmos. Sci. 25, 827-856 (1968).

${ }^{9}$ L. M. Prueitt, J. Geophys. Res. 68, 803-811, https://doi.org/10.1029/ JZ068i003p00803 (1963).

${ }^{10}$ M. A. Uman, J. Geophys. Res. 74(4), 949-957, https://doi.org/10.1029/ JB074i004p00949 (1969).

${ }^{11}$ E. P. Krinder, Nucl. Instrum. Methods 110, 411-419 (1973).

${ }^{12} \mathrm{M}$. A. Uman, IEEE Spectrum 3, 102-110 (1966).

${ }^{13}$ R. D. Hill, Rev. Geophys. 17(1), 155-164, https://doi.org/10.1029/ RG017i001p00155 (1979).

${ }^{14}$ D. Mitchard, D. Clark, D. Carr, and A. Haddad, Appl. Phys. Lett. 109, 093502 (2016).

${ }^{15}$ R. Sousa Martins, C. Zaepffel, L. Chemartin, Ph. Lalande, and A. Soufiani, J. Phys. D: Appl. Phys. 49, 415205 (2016).

${ }^{16} \mathrm{H}$. Simpson, P. Leichauer, C. Stone, and A. Haddad, in International Conference on Lighting and Static Electricity, USA (2013).

${ }^{17}$ P. Widger, A. Haddad, and H. Griffiths, IEEE Trans. Dielectr. Electr. Insul. 23(1), 14-21 (2016).

${ }^{18}$ EUROCAE WG-31 and SAE Committee AE4L, ED-84 Aircraft Lightning Environment and Waveforms Standards (EUROCAE, 1997).

${ }^{19}$ IEC 60060-1, High-Voltage Test Techniques Part 1: General Definitions and Test Requirements (IEC, 2010).

${ }^{20}$ D. Mitchard, D. Clark, D. Carr, C. Stone, and A. Haddad, J. Visualized Exp. "Method for Recording Broadband High Resolution Emission Spectra of Laboratory Lightning Arcs" (unpublished).

${ }^{21}$ D. Clark, D. Carr, D. Mitchard, C. Stone, and A. Haddad, in 20th International Symposium on High Voltage Engineering, Argentina (2017).

${ }^{22}$ A. Kramida, J. Ralchenko, J. Reader, and NIST ASD Team, NIST Database Version 5.2 (NIST, 2014). 